\title{
High-sensitive SPR sensing with Indium Nitride as a dielectric overlay of optical fibers
}

\author{
Óscar Esteban ${ }^{\mathrm{a}, *}$, Fernando B. Naranjo ${ }^{\mathrm{a}}$, Natalia Díaz-Herrera ${ }^{\mathrm{b}}$, \\ Sirona Valdueza-Felip ${ }^{a}$, María-Cruz Navarrete ${ }^{c}$, Agustín González-Cano ${ }^{b}$ \\ a Departamento de Electrónica, Universidad de Alcalá, Escuela Politécnica, 28871 Alcalá de Henares, Madrid, Spain \\ b Sección Departamental de Óptica, Escuela Universitaria de Óptica, Universidad Complutense de Madrid, Arcos de Jalón 118, 28037 Madrid, Spain \\ ${ }^{\mathrm{c}}$ Departamento de Óptica, Facultad de Ciencias Físicas, Universidad Complutense de Madrid, Ciudad Universitaria, 28040 Madrid, Spain
}

\section{A R T I C L E I N F O}

\section{Article history:}

Received 25 February 2011

Received in revised form 2 June 2011

Accepted 9 June 2011

Available online 17 June 2011

\section{Keywords:}

Tapered optical fibers

Surface Plasmon resonance

Indium Nitride

\begin{abstract}
A B S T R A C T
In this work, an Indium Nitride (InN) dielectric overlay has been used to develop a surface plasmon resonance optical fiber sensor. Although InN is a very promising material in electronics industry, this is the first time that this kind of material is used for optical sensing. The obtained results show an improvement of the reliability and long term stability with respect to previous devices made with the same technology. More remarkably, the sensitivity increases up to $11,800 \mathrm{~nm} / \mathrm{RIU}$ in the range of outer refractive indices between 1.415 and 1.429 , the highest sensitivity achieved with this kind of devices. Therefore, a novel application of the InN to optical fiber sensors is demonstrated. The use of this material would be of great interest to produce new SPR-based devices for chemical and biological sensing.
\end{abstract}

(c) 2011 Elsevier B.V. All rights reserved.

\section{Introduction}

Surface plasmon resonance (SPR) is a powerful refractometric technique that has been widely used in the field of chemical, environmental and biological sensing, becoming a mature technology in biosensing applications [1-5] where a huge number of specially designed transducers can be found in the literature [1,6-10]. Several setups have been proposed to excite surface plasmons, being the attenuated total internal reflection technique the most popular in the literature [2]. However, the use of optical fibers can simplify the experimental arrangement providing the substantial advantages offered by all-fiber intrinsic systems such are remote measurements, operation in harsh environments, easier handling, wavelength multiplexing, small size and weight $[11,12]$. Since the spectral interrogation of SPR-based sensors is the most robust technique, tunability of the resonance has been an issue in order to use the portable and low cost spectrometers based on silicon detectors.

Spectral tunability of SPR sensors, both in total internal reflection and waveguide configurations, is traditionally got with a high refractive index dielectric overlay, which is usually a metal-oxide one, deposited on top of a metallic layer [13-18], although the use of some other dielectric could be also suitable in order to achieve the desired operation point, providing that its surface can be func-

\footnotetext{
* Corresponding author.

E-mail address: oscar@depeca.uah.es (Ó. Esteban).
}

tionalized to get specific recognition of the target analyte. With this procedure, it is possible to get a specific resonance wavelength for a fixed refractive index of the outer medium. Within this approach, double-layer uniform-waist tapered fibers (DLUWTs) have been proposed in recent years to develop more compact and efficient SPR sensors [15-21].

In the past, our group has shown the advantages of adding a second layer to move the operating range of the sensors. In particular, we have shown that the adding of a second layer is mandatory to achieve SPR fiber sensing in aqueous media in the optical communications range [22]. We have also presented theoretical papers depicting the behaviour of these devices and this has also been studied by other authors [23-26].

On the other hand, it has recently been proposed the use of InN as suitable material for electronic-based biosensing due to its high superficial electron concentration [27,28]. However, this material also offers advantages in order to their use in optical biosensors. Indeed, their refractive index is larger than the obtained with the metal-oxides used up to date, mainly $\mathrm{Ta}_{2} \mathrm{O}_{5}, \mathrm{TiO}_{2}$ and $\mathrm{Al}_{2} \mathrm{O}_{3}$ [13-15], which translated to SPR sensors design implies thinner dielectric layer to get the same spectral operation point for the same refractive index of the outer medium. Furthermore, III-nitridesbased technology represents the most environmentally friendly compound semiconductor technology of the moment, which makes it very convenient for in situ environmental measurements. The specific use of InN for biosensing has been recently addressed. Several works have shown the feasibility of this material to be func- 
tionalized either with the most common functional groups [29] or with organosilane molecules [30].

InN material has been deposited using different techniques like molecular beam epitaxy (MBE), metal-organic vapour phase epitaxy (MOVPE) and sputtering. In particular, magnetron radio frequency (RF) sputtering is a commonly used technique for the deposition of nanocrystalline thin films (like metals and semiconductors) onto a substrate. Applications like optical coatings, solar cells and optoelectronic devices are based on the deposition of thin films by sputtering, verifying the interest of its research [31]. One of the main advantages is the natural simplicity of its physical process. Furthermore, sputtering enables the deposition of thin films at room temperature, and on a variety of substrates, including optical fibers and plastic substrates, making it a highly versatile low-cost technique suitable for the development of competitive sensor applications. With this technique, the deposited InN has an absorption band around $850 \mathrm{~nm}$ [32]. Thus, the excitation of surface plasmons must be done beyond this wavelength in the near infrared region.

In this work, we evaluate the performance of SPR sensors with a second InN layer deposited over the metallic one. As far as we know, this is the first time that a nitride based material is used in optical sensors, and the obtained results show a high reliability and long-term stability together with a generally good sensitivity which can be as high as $11,800 \mathrm{~nm} / \mathrm{RIU}$ in the range $1.415-1.429$ with the thinnest dielectric layer that has been tested. A really remarkable value never obtained in the past with this kind of sensors based on DLUWTs. This figure is also comparable to the highest performance of SPR sensors reported in the literature [33] and can be the starting point to the generalization of the use of DLUWTs with InN layers for biosensing.

\section{Transducers fabrication and experimental setup}

The method for obtaining uniform-waist tapered fibers has already been depicted elsewhere [17]. It is based in the travelling burner technique, where the fiber glass is heated in an oscillatory way around the length to be stretched while is gently drawn in opposite directions. With this procedure we have fabricated tapered single-mode fibers from standard silica fibers optimized for transmission at $850 \mathrm{~nm}$. The parameters of the obtained tapers are $40 \mu \mathrm{m}$ waist diameter, $12 \mathrm{~mm}$ waist length, total taper length of about $50 \mathrm{~mm}$ and losses below $0.2 \mathrm{~dB}$. On these tapers we have deposited an $\mathrm{Al} / \mathrm{InN}$ bi-layer at room temperature by RF sputtering with a 2 " confocal magnetron cathode (AJA International, ATC ORION-3-HV). This is the technique commonly used for the deposition of nanocrystalline thin films onto a substrate to develop optical coatings [34].

The aluminium layer was grown using a high purity Al target $(5 \mathrm{~N})$ in Ar plasma and $75 \mathrm{~W}$ of DC power. Using these growth conditions, a deposition rate of $2.8 \mathrm{~nm} / \mathrm{min}$ was obtained. For $\mathrm{InN}$ the target was a pure In disk (4N5) and pure $\mathrm{N}_{2}(6 \mathrm{~N})$ was used as the reactive gas. The nitride was deposited with $40 \mathrm{~W}$ of radio frequency plasma. The base pressure for the deposition was in the order of $10^{-5}$ mTorr, while the whole deposition was carried out at a working pressure of 3.5 mTorr [32]. With these parameters, we have produced several sensors, all of them with an Al layer $8 \mathrm{~nm}$ thick but with different InN thickness, namely 40, 30 and $20 \mathrm{~nm}$. The deposition procedure has been done in a static way. Thus, the obtained devices are the so-called asymmetric ones [15,18], where only approximately half of the taper is covered by the bi-layer structure with non-uniform layers thickness. A scheme of the fabricated transducers is shown in Fig. 1.

The characterization procedure for the devices has also been reported elsewhere $[15,17,18]$, and it is schematically depicted also in Fig. 1. It is based on the measurement of the spectral transmittance of the devices through an Optical Spectrum Analyzer (OSA) for different refractive indices of the outer medium, always in liquid state. Thus the procedure starts with the measurement of the transmission of the device with air as surrounding medium, being this measurement the reference for the complete characterization. Since the measurements are spectrally based, a halogen lamp has been used as light source.

Since the SPR phenomenon in asymmetric tapers is dependent on polarization, a polarization-controlling system based on a linear polarizer and a set of Lefèvre loops has been included in the setup, although the polarization is not an issue with DLUWTs, as we have shown in previous works [18]. In this case we adjust the polarization at the start of the experiments to achieve the best contrast and nothing has then to be done to prevent any further influence

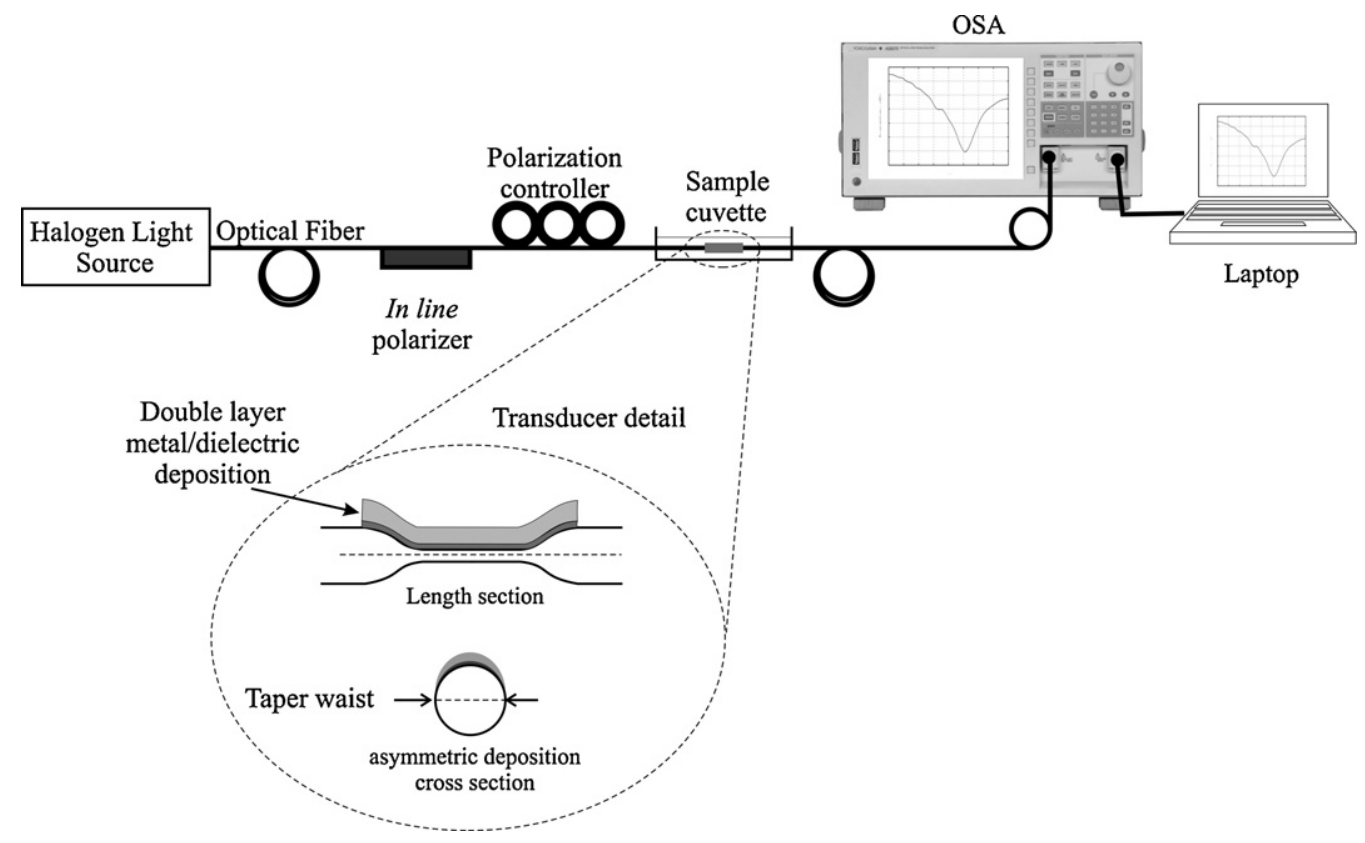

Fig. 1. Scheme of the experimental setup and detailed view of the fabricated transducers. 
in the measurements. This system should give a sharp transmittance dip when the polarization plane is aligned with the maximum thickness of the deposited layers.

In our case, the index of the outer medium can be varied continuously through either a mixture of pure water and ethylene-glycol to cover the range $1.3252-1.4089$ or pure water and glycerol to cover the range 1.4082-1.4290. The sensor is submersed in a small volume of the solution whose index is calculated from the values of the indices of water and the added ethylene-glycol or glycerol as a binary mixture [35]. In this way, it is possible to cover a wide range of refractive indices and determine their specific value for a given wavelength if the dispersion relationships of the individual components are known [36].

\section{Experimental results}

Since the main target for this kind of devices is biosensing applications, the first test was carried out with a transducer designed to resonantly excite a surface plasmon with pure water as outer medium. Using simulation routines previously developed for this kind of devices [20], it was possible to predict a resonance peak around $950 \mathrm{~nm}$, with the sensor immersed in water, when the outer dielectric layer is set to $40 \mathrm{~nm}$. Therefore, a transducer was fabricated with an InN layer $40 \mathrm{~nm}$ thick.

The measured transmittance in this case is shown in Fig. 2 with solid line, where the shortest resonance wavelength belongs to pure water (refractive index of 1.3252) as outer medium while the largest one comes from an outer refractive index of 1.3905 . In this case, the refractive index values have been calculated for the central wavelength of the investigated range, which is around $1050 \mathrm{~nm}$. With the values of the measured data, it is possible to evaluate the average sensitivity of the device around $4300 \mathrm{~nm} / \mathrm{RIU}$ from the slope of the curve plotted in Fig. 3. This value is slightly better than the reported previously in literature for DLUWTs working with $\mathrm{TiO}_{2}$ as dielectric layer in the same spectral range, which is about $3500 \mathrm{~nm} / \mathrm{RIU}$ [17].

An additional test to check the long-term stability and the reliability of the transducers was performed after one month without any special care in its conservation but a simple cover to preserve it from dust. The result of this test is also shown in Fig. 2 with dashed line. As can be seen, both measurements appear superimposed with only a small drift in some cases, which can be due to small variations in the refractive index of the measured samples. This feature is of great interest since some drift usually appeared in former SPR transducers based on tapered optical fibers with a metal-oxide as

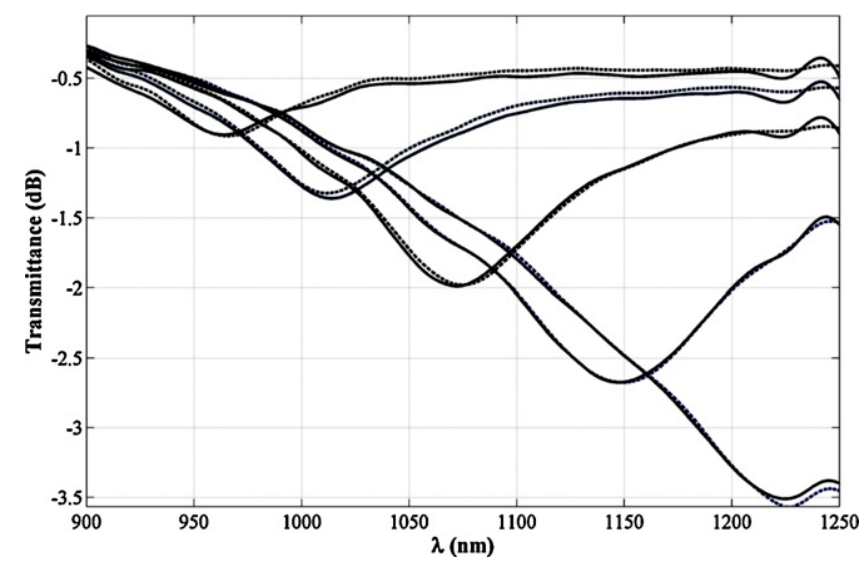

Fig. 2. Transmittance of a device with a bi-layer $\mathrm{Al} / \mathrm{InN}(8 \mathrm{~nm} / 40 \mathrm{~nm}$ thick respectively) for outer refractive indices in the range $1.3252-1.3905$ calculated at $1050 \mathrm{~nm}$. The first test is plotted in solid line while a reliability test performed one month after is plotted in dashed line.

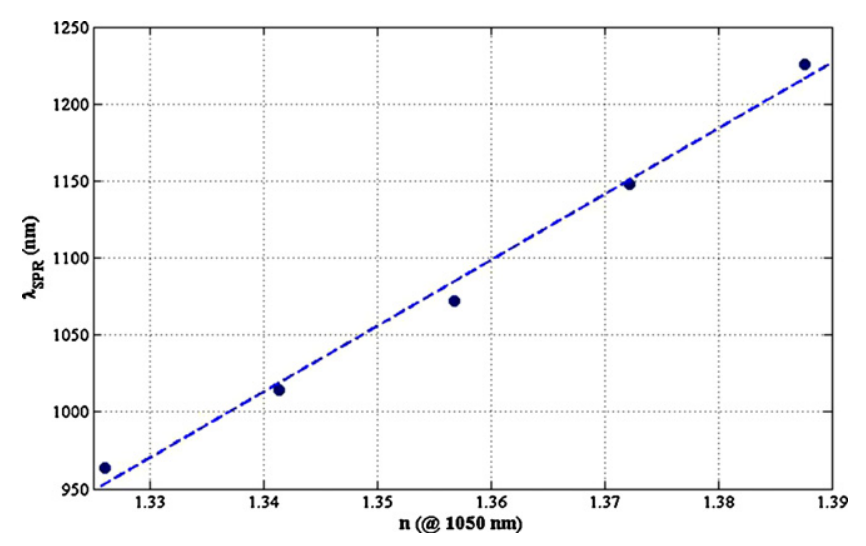

Fig. 3. Change in the resonant wavelength with the outer refractive index for the device of Fig. 2 (bi-layer $\mathrm{Al} / \mathrm{InN}, 8 \mathrm{~nm} / 40 \mathrm{~nm}$ thick respectively). The slope of the curve gives an average sensitivity around $4300 \mathrm{~nm} /$ riu.

dielectric layer. This drift was around $10 \mathrm{~nm}$ after each use, modifying the starting point of the measurement although the sensitivity was kept. With our design, the transducers become more reliable; a decisive characteristic to generalize its use as SPR based sensors.

To prove the tunability of the devices, an additional design was fabricated with an InN layer $30 \mathrm{~nm}$ thick. In this case, the characterization procedure gives a transmittance for the transducer which is shown in Fig. 4, and where the shortest resonance wavelength is obtained for an outer refractive index of 1.3950 while the largest one comes from a value of 1.4089 . Once more, the refractive index values have been estimated at the central wavelength of the range, around $900 \mathrm{~nm}$. These results are in agreement with the expected ones that predict the resonance at higher values of refractive index in the same spectral range when the thickness of the dielectric layer decreases. As it can be seen from Fig. 5, the behaviour of the response is quite linear, obtaining from the slope of the plotted curve a very high average sensitivity around $10,800 \mathrm{~nm} / \mathrm{RIU}$. This value is quite higher than the reported previously for this kind of devices.

This result is a remarkable one, since usually when the operation wavelength shifts to larger values the sensitivity increases too, as can be seen in previous results reported for SPR excitation around $1550 \mathrm{~nm}$ wavelengths [22,37], and some theoretical studies [26,38]. However, here the central wavelength of the characterized range has decreased from $1100 \mathrm{~nm}$ to $975 \mathrm{~nm}$, while the sensitivity has been doubled. Results of this kind have been depicted for different structures [39], but this is not the usual behaviour in SPR fiber sensors.

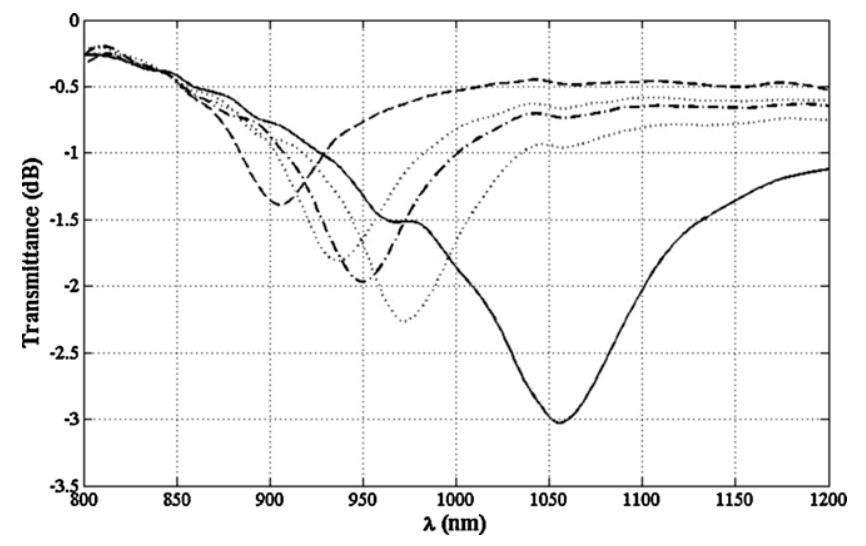

Fig. 4. Transmittance of a device with a bi-layer $\mathrm{Al} / \mathrm{InN}(8 \mathrm{~nm} / 30 \mathrm{~nm}$ thick respectively) for outer refractive indices in the range $1.3950-1.4089$ calculated at $900 \mathrm{~nm}$. 


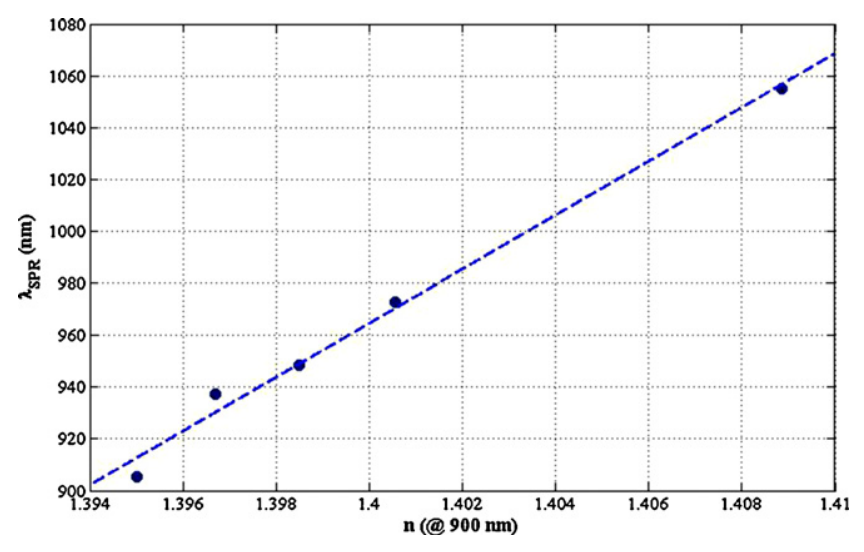

Fig. 5. Displacement of the resonant wavelength with the change of the outer refractive index for the device of Fig. 4 (bi-layer $\mathrm{Al} / \mathrm{InN}, 8 \mathrm{~nm} / 30 \mathrm{~nm}$ thick respectively). The slope of the curve renders an average sensitivity around $10,800 \mathrm{~nm} /$ riu.

Table 1

Summary of achieved sensitivities for different InN layer thickness.

\begin{tabular}{lcl}
\hline $\begin{array}{l}\text { InN thickness } \\
(\mathrm{nm})\end{array}$ & $\begin{array}{l}\text { Average sensitivity } \\
(\mathrm{nm} / \mathrm{RIU})\end{array}$ & $\begin{array}{l}\text { Refractive index range } \\
\text { (@ mean wavelength) }\end{array}$ \\
\hline 20 & 11,800 & $1.4153-1.4292(@ 1000 \mathrm{~nm})$ \\
30 & 10,800 & $1.3950-1.4089(@ 900 \mathrm{~nm})$ \\
40 & 4300 & $1.3252-1.3905(@ 1050 \mathrm{~nm})$ \\
\hline
\end{tabular}

Again the stability test was done after some time without any special care in the conservation of the fabricated transducers. The transmittance remained unchanged between several transducers fabricated with the same method while there was practically no drift in the resonance wavelength for the same values of outer refractive index. The reversibility of the measurements is complete, no changes are observed when the refractive index is decreased instead of increased. The fluctuations in the measurements are quite small, of the order of the resolution of the spectrometer, and there can exist a quite slight dependence on temperature, which only provide minor deviations in the refractive index values, below the resolution limit of the spectrometer. The stability, repeatability and reversibility of the measurements have never been issues in SPR sensing with DLUWTs and the use of InN does not change this fact.

A further device was fabricated with the InN layer $20 \mathrm{~nm}$ thick. For this transducer the central measured resonance wavelength is around $1000 \mathrm{~nm}$ (again shorter than the measured with the InN $40 \mathrm{~nm}$ thick) for the outer refractive index range from 1.4153 to 1.4292. A further increase of the outer refractive index beyond this value is not measured since the cutoff wavelength of the fiber is below the expected resonance one. In this case, the average sensitivity is around $11,800 \mathrm{~nm} / \mathrm{RIU}$, again a remarkably high value.

In Table 1, the summary of the measured sensitivity for the three devices is shown. As can be observed, it increases as the dielectric layer decreases, although, of course, the resonance that can be observed inside the detection spectral range appears for higher values of outer refractive index each case, which must be taken into account when selecting the application of the devices.

\section{Conclusions}

In this work the suitability of the use of Indium Nitride for the development of SPR-based sensors has been demonstrated. This material offers some advantages when the transducers must be functionalized in order to provide recognition of a specific analyte, since it has a high number of free nitrogen bounds on the surface that can be used to fix the recognizing elements.
A complete characterization of the dependence of the transducers behaviour with the InN thickness has been carried out, finding out that its relative high refractive index makes the layer thinner than the needed when metal-oxides like $\mathrm{Al}_{2} \mathrm{O}_{3}$ or $\mathrm{TiO}_{2}$ are used. Furthermore, the increase of the sensitivity as the InN thickness decreases has also been shown, getting sensitivity as high as $11,800 \mathrm{~nm} / \mathrm{RIU}$ when the thickness is of $20 \mathrm{~nm}$ for the range of indices between 1.415 and 1.429 , a remarkable value comparable with the most sensitive devices presented up to date. The resolution of the sensors changes between $2 \times 10^{-4}$ and $8 \times 10^{-5}$ depending on the range for a rather modest resolution of the spectrometer of $1 \mathrm{~nm}$.

Also the reliability of the transducers has been tested with measurement series at long term intervals, showing that the behaviour is stable and repeatable even without any special care in conservation of the devices. Thus the presented device could be a start up point to the generalization of the use of DLUWTs in chemical sensing and biosensing.

\section{Acknowledgements}

This work has been partially supported by Spanish Ministry of Science research projects SPRINT (reference CTQ2009-10550) and FASTCOM (reference TEC2009-14423-C02-02), by Community of Madrid project FACTOTEM II (reference S2009/ESP-1781) and by the European Social Fund and the European Fund for Regional Development.

\section{References}

[1] J. Homola, Surface plasmon resonance sensors for detection of chemical and biological species, Chem. Rev. 108 (2008) 462-493.

[2] X. Fan, I.M. White, S.I. Shopova, H. Zhu, J.D. Suter, Y. Sun, Sensitive optical biosensors for unlabeled targets: a review, Anal. Chim. Acta 620 (2008) 8-26.

[3] I. Abdulhalim, M. Zourob, A. Lakhtakia, Surface plasmon resonance for biosensing: a mini-review, Electromagnetics 28 (2008) 214-242.

[4] R. Micheletto, K. Hamamoto, S. Kawai, Y. Kawakami, Modeling and test of fiberoptics fast SPR sensor for biological investigation, Sens. Actuator A 119 (2005) 283-290.

[5] D.K. Kambhampati, W. Knoll, Surface-plasmon optical techniques, Curr. Opin. Coll. Interface Sci. 4 (1999) 273-280.

[6] A.J. Haes, R.P. Van Duyne, A unified view of propagating and localized surface plasmon resonance biosensors, Anal. Bioanal. Chem. 379 (2004) 920-930.

[7] Yong-Jun Li, Juan Xiang, Feimeng Zhou, Sensitive and label-free detection of DNA by surface plasmon resonance, Plasmonics 2 (2007) 79-87.

[8] S. Ahl, P.J. Cameron, W. Jing Liu, J. Knoll, Erlebacher, Fang Yu, A comparative plasmonic study of nanoporous and evaporated gold films, Plasmonics 3 (2008) $13-20$.

[9] N. Díaz-Herrera, Ó. Esteban, M.C. Navarrete, M. Le Haitre, A. González-Cano, In situ salinity measurements in seawater with a fibre-optic probe, Meas. Sci. Technol. 17 (2006) 2227-2232.

[10] Y.S. Dwivedi, A.K. Sharma, B.D. Gupta, Influence of design parameters on the performance of a surface plasmon sensor based fiber optic sensor, Plasmonics 3 (2008) 79-86.

[11] M. Piliarik, J. Homola, Z. Manikova, J.Čtyroký, Surface plasmon resonance sensor based on a single-mode polarization-maintaining optical fiber, Sens. Actuator B 90 (2003) 236-242.

[12] S. Tseng, K. Hsu, H. Way, K. Chen, Analysis and experiment of thin metalclad fiber polarizer wave index overlay, IEEE Photon. Technol. Lett. 9 (1997) 628-630.

[13] J. Čtyroký, J. Homola, M. Skalský, Tuning of spectral operation range of a waveguide surface plasmon resonance sensor, Electron. Lett. 33 (1997) 1246-1248.

[14] R. Alonso, F. Villuendas, J. Tornos, J. Pelayo, New in-line optical-fibre sensor basedon surface plasmon resonance excitation, Sens. Actuator A 37-38 (1993) 187-192.

[15] A. González-Cano, F.J. Bueno, Ó. Esteban, N. Díaz-Herrera, M.C. Navarrete, Multiple surface-plasmon resonance in uniform-waist tapered optical fibers with an asymmetric doublelayer deposition, Appl. Opt. 44 (2005) 519-526.

[16] Ó. Esteban, N. Díaz-Herrera, M.C. Navarrete, A. González-Cano, Surface plasmon resonance sensors based on uniform-waist tapered fibers in a reflective configuration, Appl. Opt. 45 (2006) 7294-7298.

[17] F.J. Bueno, Ó. Esteban, N. Díaz-Herrera, M.C. Navarrete, A. González-Cano, Sensing properties of asymmetric double-layer covered tapered fibers, Appl. Opt. 43 (2004) 1615-1620.

[18] M.C. Navarrete, N. Díaz-Herrera, A. González-Cano, Ó. Esteban, A polarizationindependent SPR fiber sensor, Plasmonics 5 (2010) 7-12. 
[19] A. Díez, M.V. Andrés, J.L. Cruz, In-line fiber-optic sensors based on the excitation of surface plasma modes in metal-coated tapered fibers, Sens. Actuator B 73 (2001) 95-99.

[20] J. Villatoro, D. Monzón-Hernández, E. Mejía, Fabrication and modeling of uniform-waist single-mode tapered optical fiber sensors, Appl. Opt. 42 (2003) 2278-2283.

[21] D. Monzón-Hernández, J. Villatoro, High-resolution refractive index sensing by means of a multiple-peak surface plasmon resonance optical fiber sensor, Sens. Actuator B 115 (2006) 227-231.

[22] N. Díaz-Herrera, A. González-Cano, D. Viegas, J.L. Santos, M.C. Navarrete, Refractive index sensing of aqueous media based on plasmonic resonance in tapered optical fibres operating in the $1.5 \mu \mathrm{m}$ region, Sens. Actuator B 146 (2010) 195-198.

[23] Ó. Esteban, M.C. Navarrete, A. González-Cano, E. Bernabeu, Simple model of compound waveguide structures used as fiber-optic sensors, Opt. Lasers Eng. 33 (2000) 219-230.

[24] Ó. Esteban, R. Alonso, M.C. Navarrete, A. González-Cano, Surface plasmon excitation in fiber-optic sensors: a novel theoretical approach, IEEE J. Light. Technol. 20 (2002) 448-453.

[25] Ó. Esteban, M.C. Navarrete, A. González-Cano, Theoretical method for the study of plasmon generation in hybrid multilayer-optical fibre structures, IEEE Sens. J. 5 (2005) 53-58.

[26] B.D. Gupta, A.K. Sharma, Sensitivity evaluation of a multi-layered surface plasmon resonance-based fiber optic sensor: a theoretical study, Sens. Actuator B 107 (2005) 40-46.

[27] H. Lu, W.J. Schaff, L.F. Eastman, Surface chemical modification of InN sensor applications, J. Appl. Phys. 96 (2004) 3577-3579.

[28] N. Chaniotakis, N. Sofikiti, Novel semiconductor materials for the development of chemical sensors and biosensors: a review, Anal. Chim. Acta 615 (2008) $1-9$.

[29] A. Terentjev, G. Cicero, A. Catellani, First-principles investigations of InN nonpolar surface functionalization, J. Phys. Chem. C113 (2009) 11323-11328.

[30] Chi-Fan Chen, Chung-Lin Wu, Shangjr Gwo, Organosilane functionalization of InN surface, Appl. Phys. Lett. 89 (2006) 252109.

[31] J. Wu, When group-III nitrides go infrared: new properties and perspectives, J. Appl. Phys. 106 (2009) 011101.

[32] S. Valdueza-Felip, F.B. Naranjo, M. González-Herráez, L. Lahourcade, E. Monroy, S. Fernández, Influence of deposition conditions on nanocrystalline InN layers synthesized on $\mathrm{Si}(111)$ and GaN templates by RF sputtering, J. Cryst. Growth 319 (2010) 2689-2694.

[33] R. Slavík, J. Homola, Ultrahigh resolution long range surface plasmon-based sensor, Sens. Actuator B 123 (2007) 10-12.

[34] O. Ambacher, Growth and applications of group III-nitrides, J. Phys. D: Appl. Phys. 31 (1998), 2653-.

[35] S. Sharma, P.B. Patel, R.S. Patel, J.J. Vora, Density and comparative refractive index study on mixing properties of binary liquid mixtures of eucalyptol with hydrocarbons at 303.15, 308. 15 and 313. 15 K, E-J. Chem. 4 (2007) 343349.

[36] http://refractiveindex.info/.

[37] N. Díaz-Herrera, A. González-Cano, D. Viegas, J.L. Santos, M.C. Navarrete, Ó Esteban, Moving the wavelength detection range in surface plasmon resonance sensors based on tapered optical fibers, Proc. SPIE 7653 (2010) 76531F.

[38] J. Homola, On the sensitivity of surface plasmon resonance sensors with spectral interrogation, Sens. Actuator B 41 (1997) 207-211.

[39] B. Brian, B. Sepúlveda, Y. Alaverdyan, L.M. Lechuga, Sensitivity enhancement of nanoplasmonic sensors in low refractive index substrates, Opt. Exp. 17 (2009) 2015-2023.

\section{Biographies}

Óscar Esteban received his M.Sc. degree in Physics and the Ph.D. degree in Advanced Optics from Complutense University, Madrid, Spain, in 1997 and 2001 respectively. Since 2001, he has been with the University of Alcalá, Spain, where he is currently Associate Professor in the Electronics Department and Head of the Photonics Engineering Group (GRIFO). He is also a member of the Optical Society of America (OSA) His main research subjects are optical-fiber sensors, optical testing of materials, thin solid films and optoelectronics applications.

Fernando B. Naranjo was born in Madrid, Spain, on November 4th, 1974. He received the M.S. degree in physics from Complutense University, Madrid, Spain in 1997. He received the Ph.D. degree in physics from the Polytechnic University, Madrid, Spain, in 2003. Dr. Naranjoreceived the Award of Polytechnic University at the Ph.D. Thesis in 2005.Since 2004, he is an Associate Professor at the University of Alcalá, Spain. He is a member of the Photonics Engineering Group at the University of Alcala (GRIFO), where he is currently researching on future semiconductor-based photonic devices for all optical communication networks, from deposition to device application level.

Natalia Díaz-Herrera received the M.Sc. degree in physics from the Universidad Autónoma de Madrid, Madrid, Spain, in 2001 and the Ph.D. degree in applied optics from the Universidad Complutense de Madrid (UCM) in 2005. In 2006 she joined the Optics Department of the UCM as a lecturer, where she has carried out he research and teaching activities. She has been involved in several European and Spanish projects. Her research interests are optical fiber sensors, specifically for environmental applications.

SironaValdueza-Felip received his M.Sc. degree in Telecommunication Enginee from the University Miguel Hernández in Elche (Alicante), Spain in 2006. In 2007 she joined the Photonics Engineering Group (GRIFO) of the Electronics Dept. in the University of Alcalá as research assistant to develop her Ph.D., which she expects to present in July 2011. Her research main interests are nitride materials and nonlinear optics for signal processing in telecommunication networks and optical sensing applications.

María-Cruz Navarrete received her M.Sc. degree in Physics in 1986, and her Ph.D. degree of Physics in 1994 in the Universidad Complutense de Madrid (UCM, Spain). She joined Optics Department (UCM) in 1990 where she has carried out her research and teaching activities. She has been involved in fiber optic research and in severa European and Spanish projects. During the last twelve years she has been working in optical fiber sensors, mainly applied to environmental measurements. Nowadays she is an assistant professor in the Optics Department and a member of the Applied Optics Complutense research group.

Agustín González-Cano was born in Madrid (Spain) in 1964. He received his M.Sc. in Physics (1987) and Ph.D. in Advanced Optics (1994) in the Universidad Complutense de Madrid (UCM), where he has developed his research and teaching activities during the last 22 years. Currently he is Professor in the School of Optics and Optometry of the UCM, where his main teaching subjects are Ophthalmic Optics, and Geometrical Optics. His research interests are basically optical sensors, and specifically fiber-optic sensors for environmental applications. In the past he has also been working in image processing, fringe pattern analysis and photoelasticity. In recent times he is also interested in research on the history of Optics. He has published more than 30 papers and has been involved in many different research projects, including some European Union-funded ones. He is a member of the Spanish Optics Association (SEDOPTICA). 